\title{
A Five-Year Institutional Study of the Effectiveness of Fourth-Year Clinical Anatomy in Promoting Musculoskeletal Education
}

\author{
Vandan S. Shah1, Suzanne S. Dooley-Hash², Clifford L. Craig', John L. Zeller,3,4* \\ ${ }^{1}$ Department of Internal Medicine, University of Michigan Health System, Ann Arbor, USA \\ ${ }^{2}$ Department of Emergency Medicine, University of Michigan Health System, Ann Arbor, USA \\ ${ }^{3}$ Department of Orthopedic Surgery, C.S. Mott Children's Hospital and Von Voigtlander Women's Hospital, \\ Ann Arbor, USA \\ ${ }^{4}$ Department of Medical Education, University of Michigan Medical School, Ann Arbor, USA \\ Email: ${ }^{\text {jizeller@umich.edu }}$
}

Received 9 September 2014; revised 7 October 2014; accepted 22 October 2014

Copyright (C) 2014 by authors and Scientific Research Publishing Inc.

This work is licensed under the Creative Commons Attribution International License (CC BY). http://creativecommons.org/licenses/by/4.0/

(c) (i)

Open Access

\begin{abstract}
Despite the increasing prevalence of musculoskeletal disease in the population, studies continue to show deficiencies in the duration and extent of musculoskeletal education. From 2009-2013, senior medical students enrolled in a clinical anatomy elective at our institution's medical school completed pre- and post-course examinations related to musculoskeletal education with associated radiographic interpretation and physical examination correlates. A control group of fourthyear students who did not participate in a clinical anatomy elective completed a comparable exam. Student pre-course and control group test scores averaged $54 \%$. The control group scored at the same level for the radiographic (pre-course average $65 \%$, control-group average $61 \%, p=0.21$ ) and physical exam sections (pre-course average $56 \%$, control-group average $53 \%, p=0.28$ ), and lower in the musculoskeletal portion (pre-course average $50 \%$, control-group average $39 \%, p<$ 0.001). Students completing a clinical anatomy elective scored significantly higher on their postcourse examination compared to their pre-course examination on all sections $(p<0.001)$ and scored higher than their classmates in the control group, with mean test scores of $84 \%$ for the combined sections, representing a $28 \%$ improvement. Senior medical students demonstrated deficits in knowledge related to musculoskeletal medicine. Following a focused fourth-year anatomy elective providing students with the opportunity to focus their learning of anatomy in a clinical context, students' knowledge in musculoskeletal medicine increased substantially.
\end{abstract}

${ }^{*}$ Corresponding author.

How to cite this paper: Shah, V. S., Dooley-Hash, S. S., Craig, C. L., \& Zeller, J. L. (2014). A Five-Year Institutional Study of the Effectiveness of Fourth-Year Clinical Anatomy in Promoting Musculoskeletal Education. Creative Education, 5, $1905-1912$. http://dx.doi.org/10.4236/ce.2014.521213 
Keywords

Applied Clinical Anatomy, Musculoskeletal Education, Medical Education, Anatomy Curriculum

\section{Introduction}

Musculoskeletal (MSK) injuries weigh heavily on society as it represents the most common health condition in the United States (Musculoskeletal Diseases: Leading Cause of Disability and Health Care Cost). Multiple national studies have identified that musculoskeletal diseases now constitute $20 \%$ of complaints and injuries in the emergency room (De Lorenzo, Mayer, \& Geehr, 1990) and at least 15\% of primary care visits (Pinney \& Regan, 2001). With the aging population (Public Health and Aging: Trends in Aging United States and Worldwide, 2003) and increasing prevalence of obesity, (Overweight and Obesity, 2012) future physicians will need to be adequately trained to diagnose and treat musculoskeletal conditions.

Recent studies, however, suggest that medical schools in the United States are not effectively addressing MSK medicine within their curricula (DiCaprio, Covey, \& Bernstein, 2003; Freedman \& Bernstein, 1998; Schmale, 2005). A recent study by LaPorte et al. found that only $24.7 \%$ of graduating medical students passed a validated orthopedic examination developed by Freedman and Bernstein (Skelley, Tanaka, Skelley, \& LaPorte, 2012). MSK medicine requires a solid understanding of 3-dimensional anatomy along with its clinical significance. One growing trend, however, is a reduction in time spent on human gross anatomy, one of the core foundations of medicine. The average time allocated to gross anatomy in United States allopathic medical schools has fallen from 249 hours to 144 hours from 1967 to 2001 (Gartner, 2003). The reduction raises concerns about whether the current state of anatomy education properly equips graduates for residency and beyond, (Waterston \& Stewart, 2005) especially since the first year of medical school is often the only time specifically devoted to teaching anatomy.

While many have documented the reduction in hours, investigators have not thoroughly explored the consequences and potential remedies in both the United States and the United Kingdom. A US survey attempted to quantify the adequacy of medical student preparation in gross anatomy after the significant reduction in hours. The survey investigated residency programs in family practice, emergency medicine (EM), diagnostic radiology, and general surgery regarding anatomy, its usefulness, and the quality of student preparation. The majority of program directors chose anatomy among the most important basic sciences in undergraduate medical training. In addition, $57 \%$ of program directors felt students needed a refresher in anatomy and only $29 \%$ thought students were adequately prepared (Cottam, 1999). In the UK, studies demonstrated that students who learned under a prior, more rigorous anatomy curriculum performed better on anatomy exams than students who participated in a reduced anatomy curriculum (McKeown et al., 2003).

Investigators have put forth two solutions to address the problem: the Stealth Approach and Reclaiming the Fourth Year (Bernstein et al., 2003). In the Stealth Approach, MSK medicine is woven throughout all four years of medical school, with each year reinforcing and adding to the depth of knowledge. Reclaiming the Fourth Year provides students with the opportunity of revisiting MSK medicine during fourth-year electives. In this study, we evaluated the feasibility and impact of the latter approach in improving knowledge in MSK medicine by developing and implementing two fourth-year electives in applied clinical anatomy. Student knowledge was assessed using a written examination composed of questions from the Freedman and Bernstein examination, American Association of Anatomists (AAA), and the corresponding author of this study. We hypothesized that gaps in knowledge in clinically relevant anatomy would be demonstrated among fourth-year medical students and a focused fourth-year anatomy elective would significantly improve students' knowledge.

\section{Materials and Methods}

We developed and offered two fourth-year electives at our institution's medical school: "Applied Clinical Anatomy: The Musculoskeletal System" and "Applied Clinical Anatomy: Emergency Medicine Procedures". We have offered the musculoskeletal (MSK) course for five years (2009-2013) and the emergency medicine (EM) course for four years (2010-2013). Before the start of each elective, all students took a pre-course exami- 
nation that consisted of three parts: MSK anatomy, radiographic interpretation, and physical examination correlates. The MSK and physical exam portions each consisted of 25 questions; whereas, the radiology portion consisted of 18 questions. We allotted one hour to complete each portion. The same students, upon completion of the elective, took a post-course examination with identical form at consisting of different questions based upon a similar discrimination level. Questions used on these examinations were not revealed to instructors of the course.

We also administered an examination of identical format and similar difficulty to a control group of graduating fourth-year medical students in 2011 who had not taken an anatomy elective. We recruited students by email, provided monetary compensation for their time, and informed them that we would use examination scores only for a study on anatomy education and that we would keep all personal information anonymous. In addition, we obtained student residency matches by specialty for the intervention and control groups. IRB approval was obtained (HUM00090388).

\subsection{Assessment Instrument}

Each item on our knowledge examinations required a single-best fill-in-the-blank answer that encompassed either identification of an anatomical structure/radiographic finding or the description and clinical significance of a particular physical exam correlate. Examination questions were of similar discriminatory value and randomly selected from a question bank comprised of 350 test questions incorporating those formulated by Bernstein et al., American Association of Anatomists (AAA), and Zeller et al. (Gross Anatomy, 2008; Freedman \& Bernstein, 1998) Bernstein et al. worked additionally with program directors from internal medicine residency programs and identified a passing score of $70 \%$ for their validated examination. (Freedman \& Bernstein, 2002)

\subsection{Course Design}

MSK and EM courses were comprised of morning didactic sessions followed by afternoon labs. The MSK course uses cadaver dissection with a focus on applied anatomy with clinical and radiographic correlations. It has specific focus on the execution and anatomy behind eleven common orthopedic procedures (below-knee amputation, surgical exposure for rotator cuff repair, plating of midshaft humeral fractures, surgical exposure for forearm both-bone fractures, open reduction and internal fixation of bimalleolar fractures, hip arthroplasty, knee arthroplasty, joint arthrocentesis, carpal tunnel release, cubital tunnel release, and tarsal tunnel release). The EM course has the same core foundation of knowledge but instead focuses on eleven common EM procedures performed in our simulation center (cricothyroidotomy, tracheostomy, needle throacostomy, chest tube placement, intubation, slit lamp exam, lumbar puncture, arterial line placement, central line placement, peripheral IV line, and paracentesis). These fourth-year electives approach anatomy as taught during the first year of medical school but in a more clinically orientated manner intended to significantly bolster 3-dimensional anatomy education.

\subsection{Statistical Analysis}

We compared pre-course and post-course exam scores of the intervention group using a paired t-test. We compared the control group scores to the pre-course exam scores of the intervention group using an independent groups t-test. Statistical significance was set at $p<0.05$.

\section{Results}

From 2009-2013, 49/51 (96\%) students who enrolled and completed the MSK course completed both the precourse and post-course exams. Two students did not complete pre-course exams and were thus excluded from the analysis. From 2010-2013, 50/50 (100\%) students who enrolled and completed the EM course completed both the pre-course and post-course exams. Aggregated over the two electives, 99 students completed the MSK portion, 80 students completed the radiographic interpretation portion, and 80 students completed the physical examination correlates portion. There were 19 less students taking the latter two portions because those sections were not included in the battery of exams until 2011. The control group consisted of 61 graduating seniors from our institution's medical school class of 2011. Figure 1 summarizes the performance on the knowledge test of 
the students in the control group to that of the students participating in a clinical anatomy elective, prior to and after their participation in the course.

\subsection{Control Group and Intervention Group Pre-Course Examination Performance}

The mean pre-course examination scores for the students participating in a clinical anatomy elective for the MSK, radiographic, and physical examination correlate portions were 12.5/25 (50\%), 11.6/18 (65\%), and 14.0/ $25(56 \%)$ respectively, with a cumulative mean score of 38.1/68 (56\%). Mean scores for the control group were 9.6/25 (39\%), 11.0/18 (61\%), and 13.3/25 (53\%) respectively, with a cumulative mean score of 33.9/68 (50\%). For the radiographic and physical exam portions, there were no statistically significant differences in comparing performance of the intervention and control group students $(p=0.21$ and $p=0.28$ respectively). There was a statistical difference between the two groups for the MSK portion $(p<0.001)$. The range and confidence intervals for these data are summarized in Table 1.

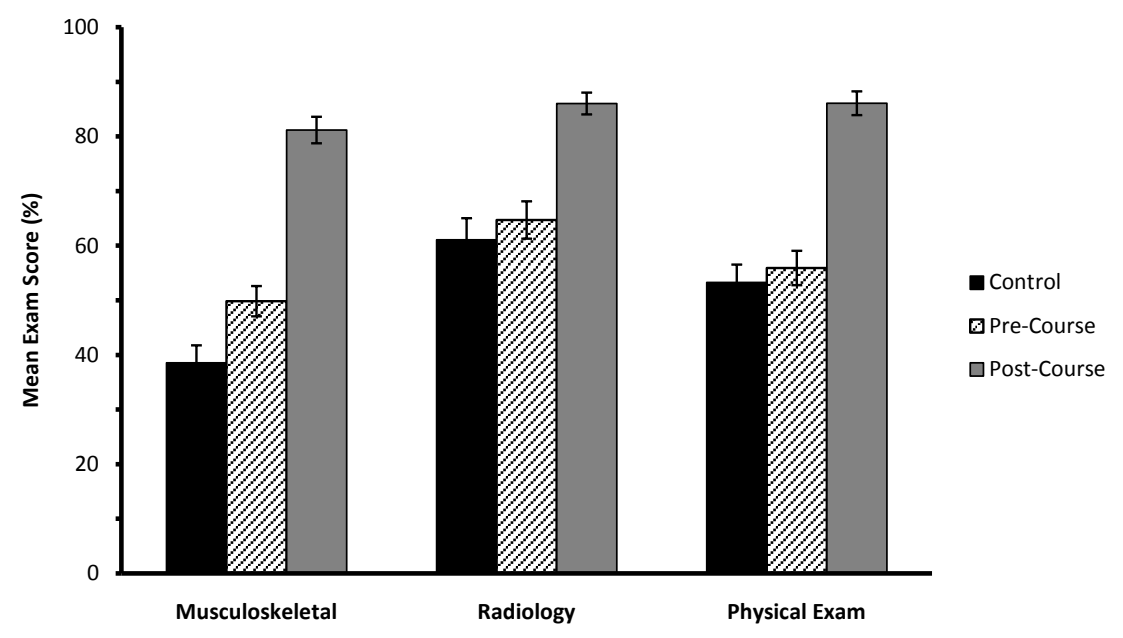

Figure 1. Exam scores for the control group and for the pre- and post-elective intervention group on the MSK anatomy, radiographic interpretation, and physical examination components among fourth-year medical students at our institution between 2009-2013. For the control group, $n=61$. For the intervention group, $n=99$ for the MSK portion and $n=80$ for the radiographic and physical examination portions. Reporting of performance scores shows mean \% correct performance, with $95 \%$ confidence intervals shown with error bars.

Table 1. Numerical values of knowledge examination scores for the control groups and for the pre- and post-elective scores of the intervention group on the MSK anatomy, radiographic interpretation, and physical examination components among fourth-year medical students at our institution between 2009-2013. For the control group, $\mathrm{n}=61$. For the intervention group, $\mathrm{n}=99$ for the MSK knowledge examination portion and $\mathrm{n}=80$ for the radiographic and physical examination portions. Reporting of performance scores shows mean $\%$ correct performance, with $95 \%$ confidence intervals shown in brackets.

\begin{tabular}{|c|c|c|c|}
\hline \multirow{2}{*}{ M4 Student Group } & \multicolumn{3}{|c|}{ Mean \% Correct Examination Score } \\
\hline & Musculoskeletal & Radiology & Physical Examination \\
\hline Control Group & $\begin{array}{c}9.6 / 25=39 \% \\
{[35-42]}\end{array}$ & $\begin{array}{c}11.0 / 18=61 \% \\
{[57-65]}\end{array}$ & $\begin{array}{c}13.3 / 25=53 \% \\
{[50-57]}\end{array}$ \\
\hline Intervention Group Pre-Course Score & $\begin{array}{c}12.5 / 25=50 \% \\
{[47-53]}\end{array}$ & $\begin{array}{c}11.6 / 18=65 \% \\
{[61-68]}\end{array}$ & $\begin{array}{c}14.0 / 25=56 \% \\
{[53-59]}\end{array}$ \\
\hline Intervention Group Post-Course Score & $\begin{array}{c}20.3 / 25=81 \% \\
{[79-84]}\end{array}$ & $\begin{array}{c}15.5 / 18=86 \% \\
{[84-88]}\end{array}$ & $\begin{array}{c}21.5 / 25=86 \% \\
{[84-88]}\end{array}$ \\
\hline$\%$ Change from Pre- to Post-Course & $+31 \%$ & $+21 \%$ & $+30 \%$ \\
\hline $\begin{array}{l}\text { Control Group vs. Intervention Group } \\
\text { Pre-Course Score }\end{array}$ & $p<0.001$ & $p=0.21$ & $p=0.28$ \\
\hline $\begin{array}{l}\text { Intervention Group Pre-Course vs. } \\
\text { Post-Course Score }\end{array}$ & $p<0.001$ & $p<0.001$ & $p<0.001$ \\
\hline
\end{tabular}




\subsection{Intervention Group Pre- and Post-Elective Examination Performance}

The mean examination scores on the post-course testing of students participating in a clinical anatomy elective for the MSK, radiographic, and physical exam correlates portions were 20.3/25 (82\%), 15.5/18 (86\%), and 21.5/ $25(86 \%)$ respectively, with a cumulative mean score of $57.3 / 68(84 \%)$. For the MSK, radiographic, and physical examination portions of the exam, the mean post-elective scores were significantly higher $(p<0.001)$ for all three sections. The magnitude of the improvement represented a $28 \%$ increase. Post-elective participation scores met and exceeded the pass score level (70\%) (Freedman \& Bernstein, 2002). The range and confidence intervals for these data are summarized in Table 1.

\subsection{Profile of Student Residency Matches}

The residency choices for both the students participating in a clinical anatomy elective and their colleagues in the control group are summarized in Table 2. Of students participating in a clinical anatomy elective, 23/99 (23\%) chose primary care specialties (Family Medicine, Internal Medicine, Internal Medicine-Pediatrics, OBGyn, Pediatrics). In the control group, 32/61 (52\%) chose primary care specialties. Outside of primary care, there was a wide distribution of residency programs in both the intervention and control groups, with the intervention group showing a large number of matches in emergency medicine and orthopedics.

\section{Discussion}

This study shows that gaps in MSK knowledge of fourth-year medical students are amenable to improvement through clinical anatomy electives. Although the number of hours of curriculum exposure provided by the fourth-year electives is less than the number of hours of anatomy curriculum in the M1/M2 years, the fourth-year electives in our study nonetheless promoted levels of improvement that were statistically significant and met and exceed recommended pass levels on the Bernstein examination (Freedman \& Bernstein, 2002).

Table 2. Distribution of residency choices for medical students in both the intervention group and control group. The intervention group consists of graduating students from our institution's medical school between 2009-2013. The control group consists of graduating students from our institution's medical school class of 2011.

\begin{tabular}{ccc}
\hline Residency & Intervention Group & Control Group \\
\hline Anesthesiology & 10 & 4 \\
Dermatology & 2 & 4 \\
Emergency Medicine & 25 & 2 \\
ENT & 2 & 7 \\
Family Medicine & 7 & 8 \\
General Surgery & 1 & 12 \\
Internal Medicine & 9 & 0 \\
Internal Medicine-Pediatrics & 5 & 3 \\
OB-Gyn & 0 & 3 \\
Ophthalmology & 0 & 0 \\
Orthopedics & 25 & 10 \\
Pediatrics & 2 & 2 \\
Psychiatry & 0 & 4 \\
Radiology & 9 & 2 \\
Urology & 2 & 61 \\
Total & 99 & 2 \\
\hline
\end{tabular}


The importance of this finding should be acknowledged in the context of the practice relevancy of MSK knowledge, given that the burden of MSK conditions on society represents a growing problem, which will be exacerbated with the aging population and increasing prevalence of obesity. Medical specialties ranging from internal medicine, family medicine, pediatrics, and emergency medicine encounter patients with MSK conditions on a daily basis, so adequate training of medical students in MSK medicine is essential. Our study demonstrates an objectively determined deficiency in senior medical students' MSK knowledge as confirmed by cumulative exam performance less than the pass score identified by recent Internal Medicine residency program directors, in both the pre-course intervention and control groups of fourth-year medical students $(56 \%$ and $50 \%$ respectively).

Exam scores from the control group and pre-course intervention group were similar for both the radiographic interpretation and physical exam correlates portions of the exam $(p=0.21$ and $p=0.28)$. We conclude students who did not take a clinical anatomy elective and those who enrolled in one of the courses had similar baseline knowledge on these areas of medicine. There was, however, a statistically significant difference $(p<0.001)$ between the two groups with regards to the musculoskeletal anatomy portion of the exam, with higher scores for the intervention group $(50 \%)$ than the control group $(39 \%)$. This portion of the exam addressed structural anatomy in a clinical context. The difference between the student groups likely reflects a greater inclination towards anatomical knowledge for those students taking a clinical anatomy elective. However, pre-course examination scores from the intervention group and in the control group revealed knowledge deficits with performances below recommended passing levels (Freedman \& Bernstein, 2002).

The similar low performance in the intervention and control groups is indicative of the knowledge base of MSK medicine of M4 students at our institution. Other medical schools have documented a lack of knowledge among fourth-year students in these same areas. Bernstein et al. showed that seventy of eighty-five (82\%) recent medical school graduates from their medical school failed to meet passing standards, set by 157 chairpersons of orthopedic residency programs, on an MSK competency exam (Freedman \& Bernstein, 1998). Sixty-six students (78\%) failed the exam when passing standards were set by 244 program directors of internal medicine residency (Freedman \& Bernstein, 1998, 2002). Studies by LaPorte et al. found $75 \%$ of fourth-year medical students at their respective institution failed the original Freedman and Bernstein examination (Skelley et al., 2012).

After completing a clinical anatomy elective, students showed a significant improvement in their examination performance. For all three sections of the exam (MSK anatomy, radiologic interpretation, and physical examination correlates), the mean score increased by 20 percentage points or higher $(p<0.001)$. Similar results were obtained by LaPorte et al. who showed that students who participated in elective musculoskeletal education had a higher pass rate (67.5\%) than those who did not (43.9\%) (Skelley et al., 2012). Even students entering more anatomy-focused fields such as orthopedics and EM had initial deficits in understanding MSK pathology. After completing either clinical anatomy elective, however, students met or exceeded recommended pass scores in all three areas. These students, we feel, are well equipped to enter their residency with a solid foundation in clinical anatomy and are better suited to address MSK conditions in their future practices.

One limitation of the study involves the composition of the intervention group, with a majority of those individuals entering either orthopedic surgery or EM $(50 / 99,51 \%)$. These students may have had more initial interest in MSK medicine and thus gained more knowledge from the courses than students not selecting either clinical anatomy elective. Although students in the intervention group had inclinations towards anatomically oriented programs, there were, however, a number of individuals entering primary care specialties $(23 / 99,23 \%)$. This is in contrast to the composition of the control group, whose range of specialties concentrated in internal medicine $(12 / 51,24 \%)$, pediatrics $(10 / 51,20 \%)$, and family medicine $(7 / 51,14 \%)$.

Second, this is a single institutional study. It depicts the experience at our institution's medical school and may not be representative of students at other institutions, especially with the wide variety of medical school curriculums. However, the length of time that our medical school allocates to MSK education two weeks during the second year-represents the national mean hours devoted to MSK medicine (DiCaprio et al., 2003). Thus, we feel our institution does not emphasize MSK education any more or less than other institutions.

Revisiting anatomy during the fourth year provides the advantage of teaching students who have significant clinical experience. First and second-year students lack the exposure to clinical medicine compared to fourthyear students. Thus, senior students are likely better able to understand the relevance of the anatomy. Having an understanding of the relevance is one of the 10 principles of effective clinical learning identified by Rofle and Sanson-Fisher (Rolfe \& Sanson-Fisher, 2002). 
Teaching in context is not a new idea. The context used for teaching anatomy can be divided into four categories: clinical skills (physical examination), pathology (cancer, neurological diseases, musculoskeletal problems), radiology, and surgical procedures (Bergman, van der Vleuten, \& Scherpbier, 2011). Our MSK and EM electives directly address these four areas. In our two clinical anatomy electives, anatomy was taught as it pertained to understanding physical examinations, diagnosing and treating musculoskeletal injuries, interpreting radiographic images, and carrying out common medical procedures. These skills are invaluable to a medical student about to enter his or her internship. Culminating all the clinical skills acquired in four years and revisiting salient points in context should help promote MSK education.

\section{Conclusion}

In summary, we see fourth-year electives as a solution to multiple problems. It bolsters anatomy education in a clinically significant way, prepares students about to begin their residency, and provides training in important areas of medicine. Very few articles have investigated a vertical integration of anatomy (Bergman et al., 2011). There is much potential benefit in fourth-year clinical anatomy electives. Adding such electives can bolster anatomy and MSK education without significantly disrupting the current curriculum.

\section{Acknowledgements}

The authors wish to thank The Center for Statistical Consultation and Research (CSCAR) at our institution for assistance with the statistical analysis. We gratefully acknowledge the efforts and assistance provided by Dean A. Mueller, BMS, MOL (Director of Anatomical Donations Program at our institution).

\section{References}

Bergman, E. M., van der Vleuten, C. P., \& Scherpbier, A. J. (2011). Why Don't They Know Enough about Anatomy? A Narrative Review. Medical Teacher, 33, 403-409.

Bernstein, J., Alonso, D. R., DiCaprio, M., Friedlaender, G. E., Heckman, J. D., \& Ludmerer, K. M. (2003). Curricular Reform in Musculoskeletal Medicine: Needs, Opportunities, and Solutions. Clinical Orthopaedics and Related Research, 415, 302-308. http://dx.doi.org/10.1097/01.blo.0000093922.26658.3c

Cottam, W. W. (1999). Adequacy of Medical School Gross Anatomy Education as Perceived by Certain Postgraduate Residency Programs and Anatomy Course Directors. Clinical Anatomy, 12, 55-65. http://dx.doi.org/10.1002/(SICI)1098-2353(1999)12:1<55::AID-CA8>3.0.CO;2-O

De Lorenzo, R. A., Mayer, D., \& Geehr, E. C. (1990). Analyzing Clinical Case Distributions to Improve an Emergency Medicine Clerkship. Annals of Emergency Medicine, 19, 746-751. http://dx.doi.org/10.1016/S0196-0644(05)81697-X

DiCaprio, M. R., Covey, A., \& Bernstein, J. (2003). Curricular Requirements for Musculoskeletal Medicine in American Medical Schools. The Journal of Bone and Joint Surgery (American Volume), 85-A, 565-567.

Freedman, K. B., \& Bernstein, J. (1998). The Adequacy of Medical School Education in Musculoskeletal Medicine. The Journal of Bone and Joint Surgery (American Volume), 80, 1421-1427.

Freedman, K. B., \& Bernstein, J. (2002). Educational Deficiencies in Musculoskeletal Medicine. The Journal of Bone and Joint Surgery (American Volume), 84-A, 604-608.

Gartner, L. P. (2003). Anatomical Sciences in the Allopathic Medical School Curriculum in the United States between 19672001. Clinical Anatomy, 16, 434-439. http://dx.doi.org/10.1002/ca.10159

McKeown, P. P., Heylings, D. J., Stevenson, M., McKelvey, K. J., Nixon, J. R., \& McCluskey, D. R. (2003). The Impact of Curricular Change on Medical Students' Knowledge of Anatomy. Medical Education, 37, 954-961. http://dx.doi.org/10.1046/j.1365-2923.2003.01670.x

(2013). Musculoskeletal Diseases: Leading Cause of Disability and Health Care Cost.

(2012). Overweight and Obesity. http://www.cdc.gov/obesity/data/adult.html

Pinney, S. J., \& Regan, W. D. (2001). Educating Medical Students about Musculoskeletal Problems. Are Community Needs Reflected in the Curricula of Canadian Medical Schools? The Journal of Bone and Joint Surgery (American Volume), 83-A, 1317-1320.

(2003). Public Health and Aging: Trends in Aging_-United States and Worldwide. http://www.cdc.gov/mmwr/preview/mmwrhtml/mm5206a2.htm

Rolfe, I. E., \& Sanson-Fisher, R. W. (2002). Translating Learning Principles into Practice: A New Strategy for Learning Clinical Skills. Medical Education, 36, 345-352. http://dx.doi.org/10.1046/j.1365-2923.2002.01170.x 
Schmale, G. A. (2005). More Evidence of Educational Inadequacies in Musculoskeletal Medicine. Clinical Orthopaedics and Related Research, 437, 251-259. http://dx.doi.org/10.1097/01.blo.0000164497.51069.d9

Skelley, N. W., Tanaka, M. J., Skelley, L. M., \& LaPorte, D. M. (2012). Medical Student Musculoskeletal Education: An Institutional Survey. The Journal of Bone and Joint Surgery (American Volume), 94, e146 (1-7).

Waterston, S. W., \& Stewart, I. J. (2005). Survey of Clinicians' Attitudes to the Anatomical Teaching and Knowledge of Medical Students. Clinical Anatomy, 18, 380-384. http://dx.doi.org/10.1002/ca.20101 
Scientific Research Publishing (SCIRP) is one of the largest Open Access journal publishers. It is currently publishing more than 200 open access, online, peer-reviewed journals covering a wide range of academic disciplines. SCIRP serves the worldwide academic communities and contributes to the progress and application of science with its publication.

Other selected journals from SCIRP are listed as below. Submit your manuscript to us via either submit@scirp.org or Online Submission Portal.
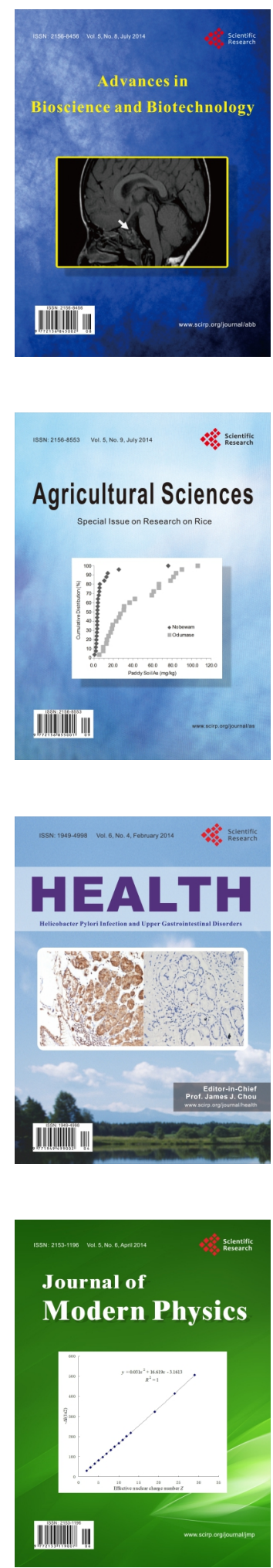
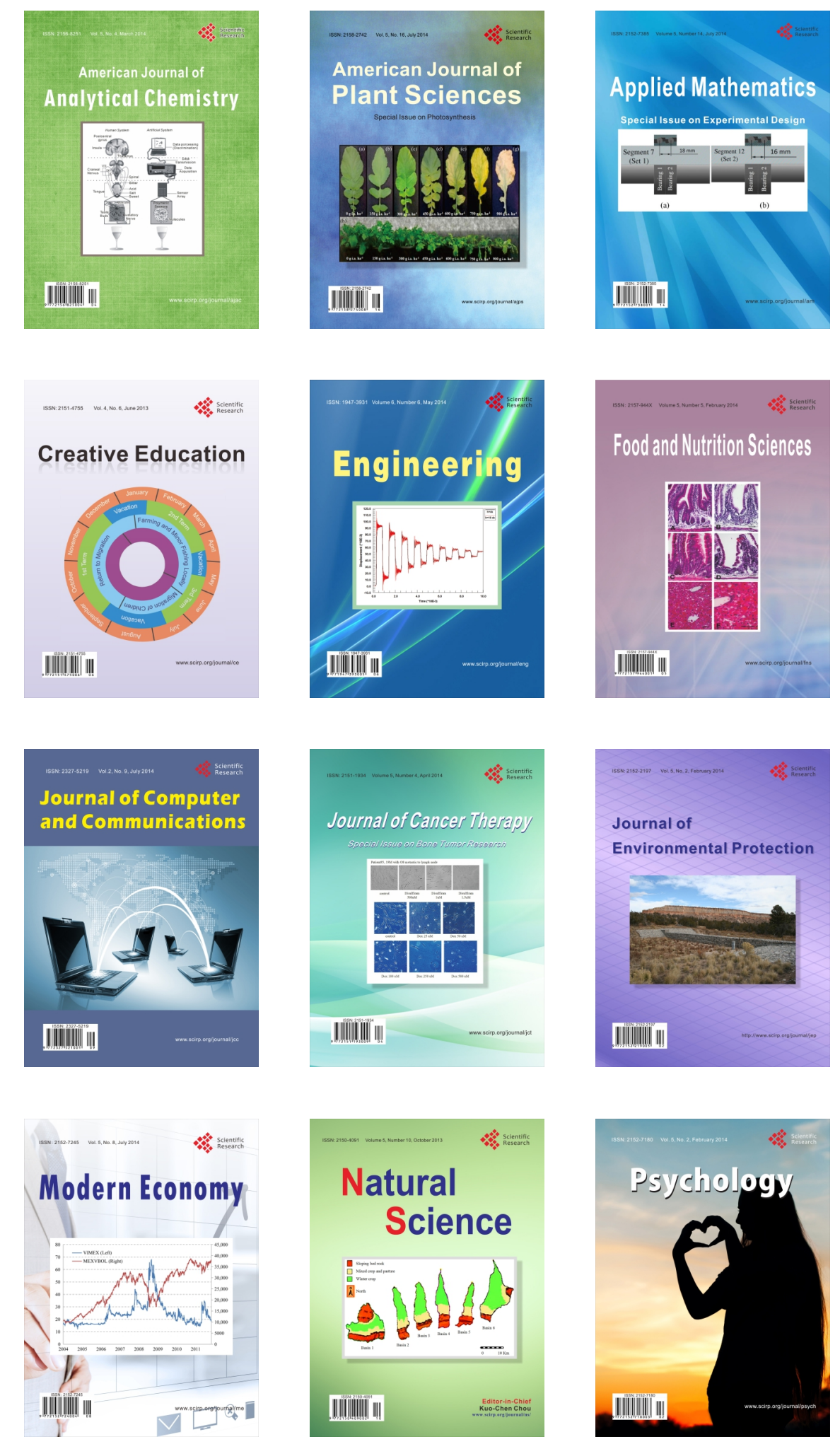